\title{
A Wideband Waveguide Antenna with Nearly Equal E- and H-Plane Radiation Patterns
}

\author{
Mei Qing Qi, ${ }^{1,2}$ He-Xiu Xu, ${ }^{3}$ Hui Feng Ma, ${ }^{1}$ Mou Ping Jin, ${ }^{2}$ Wei Wang, ${ }^{2}$ and Tie Jun Cui ${ }^{1}$ \\ ${ }^{1}$ School of Information Science and Engineering, Southeast University, 2 Sipailou, Jiangsu, Nanjing 210096, China \\ ${ }^{2}$ East China Research Institute of Electronic Engineering, 199 Xiangzhangdadao, Anhui, Hefei 230088, China \\ ${ }^{3}$ Department of Electromagnetic Field and Microwave Technology, Missile Institute of Air Force Engineering University, \\ Shanxi, Xian 710051, China \\ Correspondence should be addressed to Tie Jun Cui; tjcui@seu.edu.cn
}

Received 13 October 2012; Revised 29 December 2012; Accepted 30 December 2012

Academic Editor: Tat Yeo

Copyright (c) 2013 Mei Qing Qi et al. This is an open access article distributed under the Creative Commons Attribution License, which permits unrestricted use, distribution, and reproduction in any medium, provided the original work is properly cited.

\begin{abstract}
A novel wideband waveguide antenna with excellent performance is proposed, which is composed of a coaxial-waveguide transition and an open-ended rectangular waveguide loaded with a pair of horizontal umbrella-shaped metallic brims. The brims perpendicular to two broad walls of the waveguide are constructed to modulate the antenna to radiate nearly identical E- and $\mathrm{H}$ plane radiation patterns. A wideband impedance matching performance is achieved with a fractional bandwidth of $40 \%$ through the introduction of short-stepped ladders. The antenna has the advantages of simple structure, symmetric radiation pattern, low-cross polarization, moderate back lobe, and almost constant beamwidth.
\end{abstract}

\section{Introduction}

The demand for the wideband, low-profile antennas with stable gain as well as symmetrical radiation patterns is on the rise with the fast growth of microwave and millimeter-wave wireless communication systems. Up to date, the pursuing of these high-performance antennas has attracted much attention and long-held interest. Microstrip patch antennas exhibiting the advantages of low profile, light weight, low cost, easy fabrication, and high integration with other devices have been widely adopted for electric systems. However, their impedance bandwidths are not sufficient, and also the gains are impossible to remain stable across a broad frequency range, which restricts considerably the practical applications. Although some impedance bandwidth enhancement techniques have been developed such as multilayer structure [1], U-slot loading [2], and L-probe feeding [3], the stability of radiation patterns are essentially difficult to be held. Some modified planar monopole antennas $[4,5]$ have been proposed with ultrawide bandwidths but they still do not satisfy the rigorous requirement because of their unstable radiation patterns. Other wideband antennas including the planar spiral, log-periodic, Vivaldi, Yagi antenna, and double-ridged horn have been implemented after a survey of available literature. However, they are apparently with the drawback of large profile or complicated configurations.

Dipole is a fundamental radiator and is often used as an equivalent source in the analysis of antenna. It is evident that unequal radiation patterns are achieved in the two principal planes for both an electric dipole and a magnetic dipole. This is because interchanged radiation patterns of figure-o and figure- 8 are obtained between both dipoles. To implement equal $\mathrm{E}$ - and $\mathrm{H}$-plane radiation patterns with low back radiation, Clavin and his coworkers presented the idea of complementary antennas $[6,7]$ where nearly equal E- and $\mathrm{H}$-plane radiation patterns were achieved by incorporating an electric dipole and a magnetic dipole with appropriate amplitude and phase. Recently, Luk et al. advanced a step forward who were enlightened by the concept of magnetoelectric dipole and proposed a series of wideband unidirectional antennas with stable gain. In their design, the antennas were mainly composed of a vertically oriented quarter-waveshorted patch and a horizontal planar dipole excited by $\Gamma$ shaped strip probes $[8,9,10,11]$ or a coaxial feed [12]. Of them, however, some structures are somewhat complicated especially for their feeding which is typically associated with 


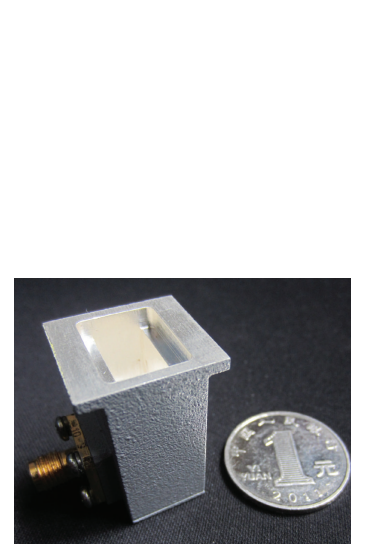

(a)

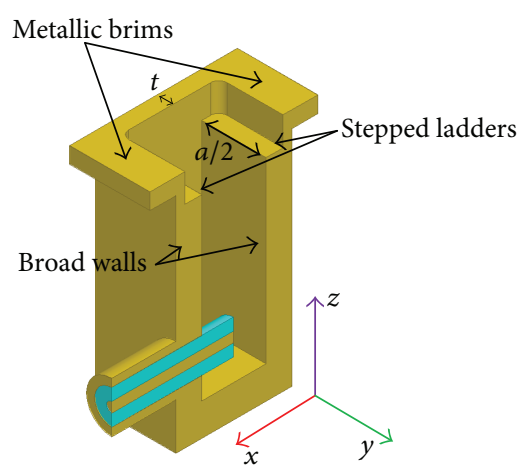

(b)

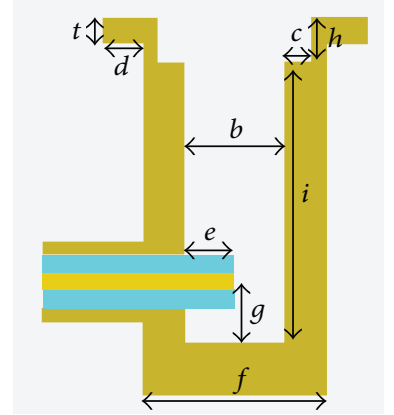

(c)

FIGURE 1: The fabricated prototype and topology of the proposed antenna. (a) The photograph of the prototype antenna. (b) The cut-open view of the proposed antenna. (c) The cross-section along E-plane of the proposed antenna. The detailed dimensions (unit: $\mathrm{mm}$ ) are $a=15.8$, $b=7.9, c=2.1, d=2.7, e=3.9, f=14.3, g=4.85, h=3.5, i=22$, and $t=2$.

a complicated design and thus may be hard to be popularized into practical applications, making an alternative, improved strategy a challenging and pressing task.

In this paper, a simplified feed is introduced to excite the complementary antenna which is evolved from the openended rectangular waveguide antenna. Unlike the complicated corrugated conical horns [13], the proposed antenna not only overcomes the drawback of conventional openended rectangular waveguide antenna whose beamwidth in E-plane is usually unequal to that in H-plane [14] but also is with very simple structure which is often related with an easy design and fabrication. It thus should be a good candidate in the modern wireless and satellite communication systems. In addition, due to its unique radiation characteristics, the proposed antenna can also be well applied to illuminate a circular aperture such as circular paraboloid [6] or lens.

\section{Antenna Structure and Design}

2.1. Geometry of the Waveguide Antenna. Figure 1 illustrates the structure and the geometrical dimensions of the proposed waveguide antenna which consists of an open-ended rectangular waveguide, stepped ladders along the broad walls, and umbrella-shaped metallic brims. Notice that beamwidth of the conventional open-ended rectangular waveguide in E-plane is wider than that in H-plane. To obtain an equal E-plane and $\mathrm{H}$-plane radiation pattern, a complementary antenna with beamwidth in E-plane narrower than that in H-plane is necessary. For this purpose, the rectangular waveguide is loaded with a pair of horizontal umbrellashaped metallic brims which functions as a planar electric dipole whose beamwidth in E-plane is narrower than that in H-plane (see Figure 4). In the present work, the antenna is designed to operate in the whole $\mathrm{Ku}$-band ranging from $12 \mathrm{GHz}$ to $18 \mathrm{GHz}$. The geometrical parameters are optimized and detailed in Figure 1, in which $a$ and $b$ are the length and width of the waveguide cross-section, $c$ and $h$ are the height and length of the stepped ladder, and $t$ in conjunction with $d$ are the thickness and length of the metallic brims, respectively.

The rectangular waveguide antenna is simply excited by a coaxial probe with the diameter of $1.27 \mathrm{~mm}$, which is accompanied by a dielectric and a coaxial-waveguide transition. The dielectric in the SMA connector covering the probe has a relative permittivity of $\epsilon_{r}=2.08$. The metal that is utilized in the design and fabrication is copper with the conductivity of $\sigma=5.8 \times 10^{7} \mathrm{~S} / \mathrm{m}$, and silver is adopted to cover the surface inside the waveguide to reduce the transmission loss.

2.2. Input Impedance Matching Performance. As an antenna, a conventional open-ended rectangular waveguide cannot be well matched to free space over the entire operating frequency band [15]. Hence extensive simulations have been performed, which demonstrate that the impedance bandwidth of the waveguide antenna is determined by two factors. The first factor is the transition from coaxial to rectangular waveguide. The second is the reflection at the end of the waveguide which exhibits a capacitive reactance. To show the corresponding result, the relative input impedance of conventional openended WR62 waveguide on a Smith chart obtained from commercial EM full-wave simulator HFSS is shown in Figure 2. As can be seen, an obvious capacitive reactance is confirmed. Moreover, the real part of input impedance approximates to unity. According to the transmission line theory, the complex input impedance including the real (resistive) and imaginary (reactive) components can be transformed to a pure resistive one through a portion of transmission line with appropriate length. In this particular case, a pair of stepped ladders along the broad walls is introduced, and the length $h$ of them is selected about one eighth of the waveguide wavelength $\left(\lambda_{g}\right)$ corresponding to the center frequency $15 \mathrm{GHz}$ to cancel out the capacitive reactance. Moreover, the stepped ladders 


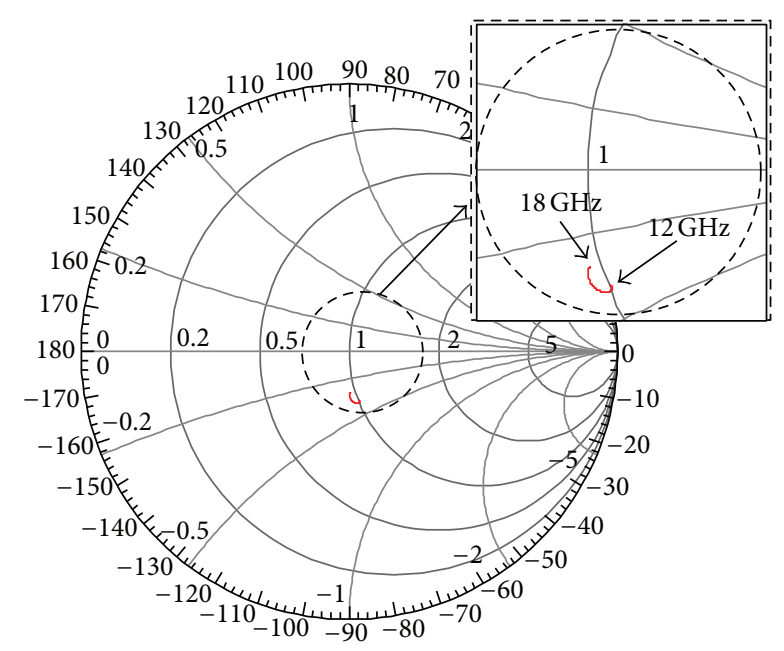

FIGURE 2: Simulated relative input impedance of conventional openended WR62 waveguide without ladders within entire Ku-band.

considerably counteract the resulting capacitive reactance in a broad band.

To corroborate the elucidation, Figure 3 depicts the simulated voltage standing-wave ratio (VSWR) against the frequency as a function of $h$ (see Figure 3(a)) and $c$ (see Figure $3(\mathrm{~b})$ ), respectively. It is clearly observed that the VSWR of the designed antenna decreases as $h$ increases from zero to $3.5 \mathrm{~mm}$ stepped by $1.75 \mathrm{~mm}$ ( $c$ is fixed as $2.1 \mathrm{~mm}$ ). Remark that the case $h=0 \mathrm{~mm}$ corresponds to the open-ended waveguide antenna without ladders. From Figure 3(b), it can be clearly observed that the VSWR of the designed antenna does not always reduce as $c$ increases ( $h$ is fixed as $3.5 \mathrm{~mm}$ ); instead, it initially reduces and then deteriorates, which demonstrates that the introduced stepped ladders play an important role in determining the matching performance of the antenna and are able to improve the matching providing an appropriate dimension. In fact, the VSWR is minimum around $c=1.2 \mathrm{~mm}$ and is below 1.2 over the entire band. In the final fabricated prototype, the value of $c$ is selected as $2.1 \mathrm{~mm}$ to consider the tradeoff between the impedance match and the equality of the beamwidths in E-plane and $\mathrm{H}$ plane within the entire Ku-band. In the finally implemented antenna ( $h=3.5 \mathrm{~mm}$ (nearly $\lambda_{g} / 8$ at $15 \mathrm{GHz}$ ) and $c=$ $2.1 \mathrm{~mm}$ ), numerical results indicate that the VSWR is better than 1.4 across the whole Ku-band.

\subsection{Radiation Characteristics. To investigate the operation} mechanism of the proposed waveguide antenna, the surface current distributions of the antenna excited in the case of different phases $(\phi)$ and the inside surface current of $\mathrm{TE}_{10}$ mode along the broad and narrow wall of the waveguide are demonstrated in Figure 4. From the inside surface current distribution of the dominant mode $\mathrm{TE}_{10}$, we obtain that the longitudinal components and the transverse ones change alternately. In the case of $\phi=0^{\circ}$, the transverse components of inside surface current of $\mathrm{TE}_{10}$ mode on the aperture are dominative while the longitudinal ones are minimized (see Figure 4(e)). The aperture current mainly concentrates around the aperture and forms a circular loop with two components flowing along opposite directions, resembling a "one-wavelength" loop antenna. On the contrary, in the case of $\phi=90^{\circ}$, the current along the horizontal umbrella-shaped metallic brims is up to the maximum while the one around the aperture is minimized, corresponding to the case when longitudinal current of $\mathrm{TE}_{10}$ mode is dominative. Therefore, an electric dipole has been effectively excited as claimed in the previous section. Similar to the first case, the current around the aperture at $\phi=180^{\circ}$ is dominative; however, its direction is opposite to that in the case of $\phi=0^{\circ}$. In the case of $\phi=270^{\circ}$, the electric dipole is excited again, and the resulting current direction is opposite to that in the case of $\phi=90^{\circ}$. In summary, the proposed antenna is capable of exciting a complementary antenna by means of alternately exciting aperture current along two orthogonal directions.

From the observation of the operation mechanism of the proposed antenna, we can conclude that the current distribution on the aperture is mainly determined by the geometrical shape of the aperture. Although the distribution of the inside surface current for the $\mathrm{TE}_{10}$ mode of rectangular waveguide is determined when its transverse size is given, we still can control the aperture current distribution by changing the shape of the aperture. In this case, it is particularly interesting to study the relation between the dimensions of metallic brims and radiation performance of the antenna. The study would afford us a useful guideline in the design of the waveguide antenna with equal E- and H-plane radiation patterns by exciting simultaneously the complementary antenna with appropriate amplitude and phase. Figure 5 illustrates the simulated beamwidth in $\mathrm{E}$ - and $\mathrm{H}$-planes as a function of $d$. We remark that $d=0 \mathrm{~mm}$ corresponds to the conventional waveguide antenna without brims. Following the figure, it is evident that nearly equal $\mathrm{E}$ - and $\mathrm{H}$-plane radiation patterns are engineered at three selected representative frequencies when $d=$ $2.7 \mathrm{~mm}$. However, the uniform radiation patterns degrade to a certain degree when $d$ takes other values. Particularly, in the condition when $d$ takes zero, the beamwidth in E-plane is much wider than that in $\mathrm{H}$-plane, implying a large discrepancy of the radiation patterns. The mechanism can be simply explained as follows: the variation of $d$ affects the amplitude and phase of the equivalent electric dipole. To obtain identical radiation patterns in the two principal radiation planes, $d$ is chosen as $2.7 \mathrm{~mm}$ in the final design and fabrication.

Note that the width of the brim is selected equal to that of the waveguide in the above analysis. For comprehensive study, extensive simulations have also been performed in the case when $y$-directed brims (perpendicular to the narrow walls of the waveguide) are introduced. For analysis convenience, the length of the $y$-directed brims that extends the waveguide is defined as $d_{y}$. Not to lose generality, a typical value of $d_{y}=3 \mathrm{~mm}$ is selected. The corresponding simulated results are also afforded in Figure 3 and Table 1. As can be seen, the VSWR is nearly invariable, and the gain is slightly increased when brims are introduced in $y$ direction. Moreover, the back radiation levels change slightly. However, the beamwidths in E- and H-planes are decreased considerably. To illustrate the effect of the length of the metallic brims $d$ on the radiation performance, Figure 6 portrays 


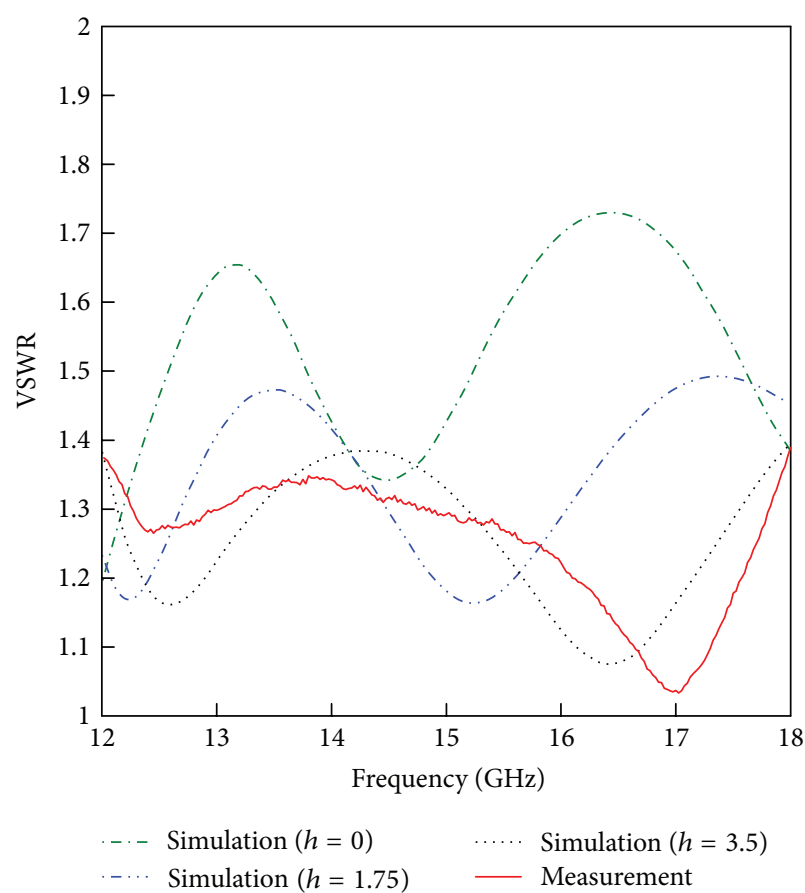

(a)

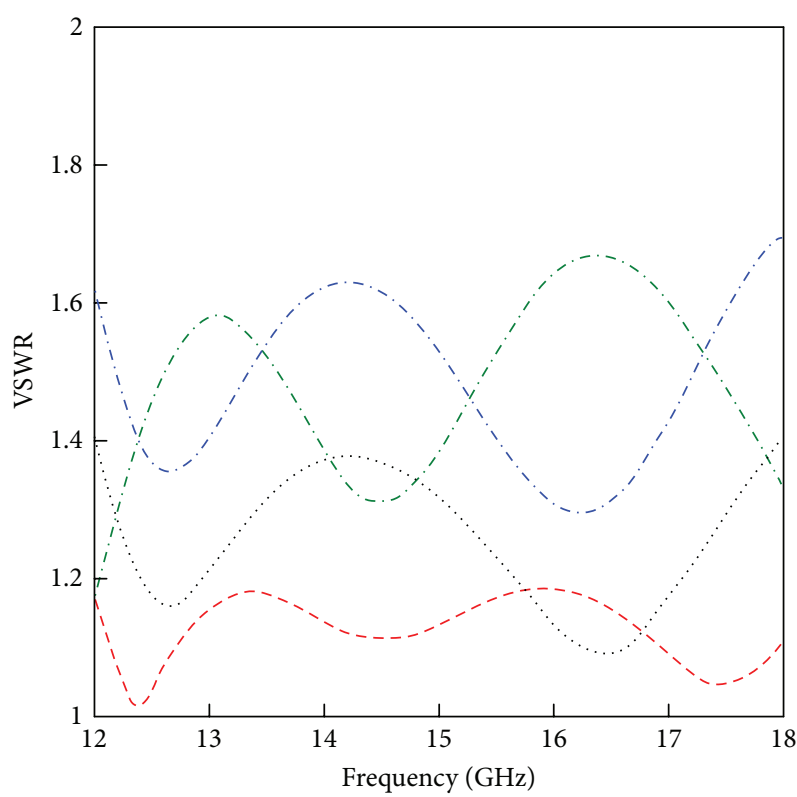

Simulation

$\cdot-c=0 \mathrm{~mm}$ $\begin{aligned}-c & =3 \mathrm{~mm}\end{aligned}$

(b)

FIGURE 3: Simulated and measured VSWRs of the proposed antenna versus the dimensions of the stepped ladders (a) as a function of $h$, (b) as a function of $c$.

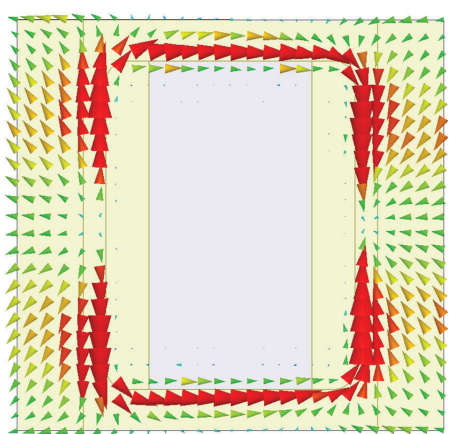

(a)

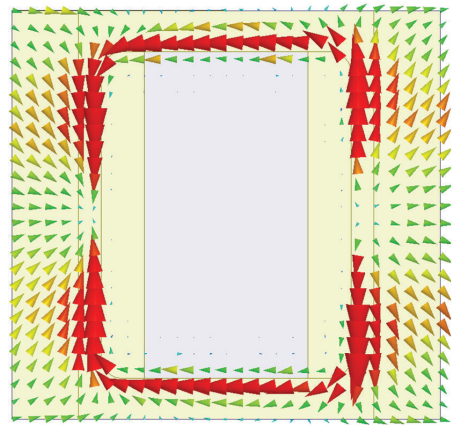

(c)

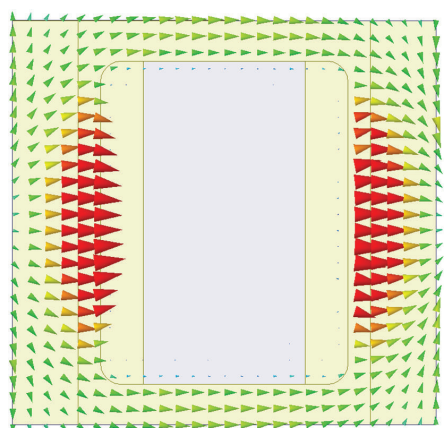

(b)

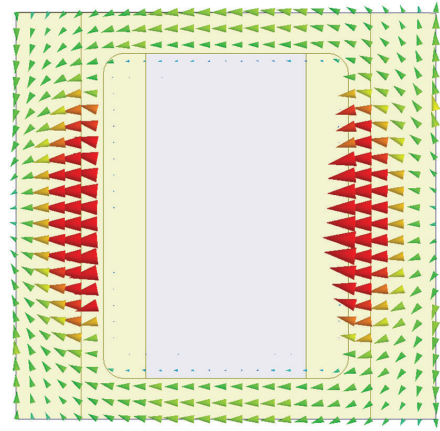

(d)
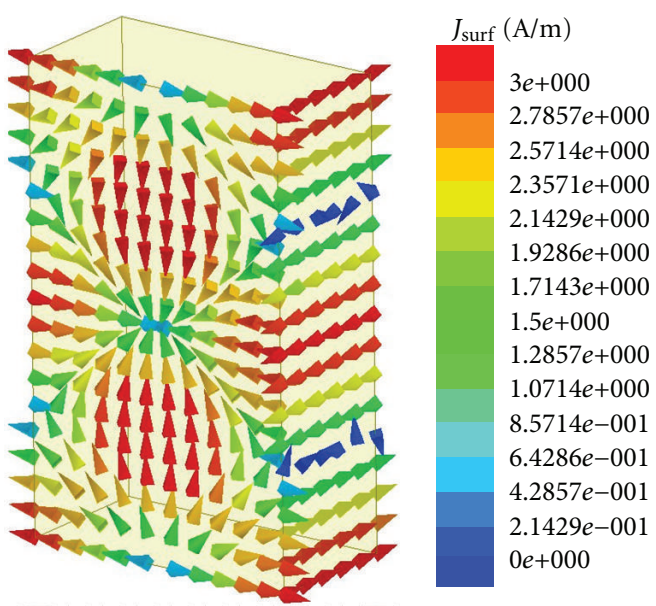

(e)

FIGURE 4: Surface current distributions of the proposed antenna in the case of different excitation phases. Aperture current distributions in the case of (a) $\phi=0^{\circ}$; (b) $\phi=90^{\circ}$; (c) $\phi=180^{\circ}$; (d) $\phi=270^{\circ}$; and (e) inside surface current of $\mathrm{TE}_{10}$ mode in the case of $\phi=0^{\circ}$. 


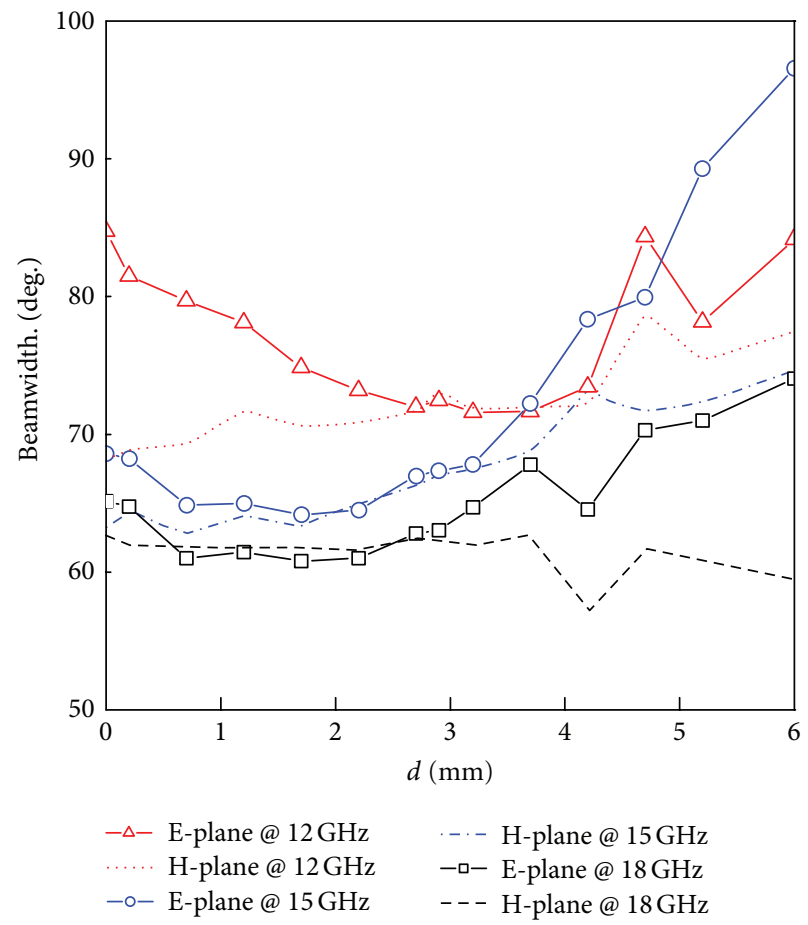

FIgURE 5: Comparison of beamwidths in E- and H-planes as functions of $d$.

TABLE 1: Simulated and measured 3-dB beamwidths and gains of the proposed antenna.

\begin{tabular}{|c|c|c|c|c|c|c|}
\hline \multirow{3}{*}{$\begin{array}{l}\text { Freq. } \\
(\mathrm{GHz})\end{array}$} & \multicolumn{3}{|c|}{ 3-dB beamwidth (deg.) } & \multicolumn{3}{|c|}{ Gain $(\mathrm{dBi})$} \\
\hline & \multicolumn{2}{|c|}{ Simulation } & \multirow{2}{*}{$\begin{array}{l}\text { Measurement } \\
\text { E-plane/H-plane } \\
\quad\left(d_{y}=0 \mathrm{~mm}\right)\end{array}$} & \multicolumn{2}{|c|}{ Simulation } & \multirow{2}{*}{$\begin{array}{l}\text { Measurement } \\
d_{y}=0 \mathrm{~mm}\end{array}$} \\
\hline & $\begin{array}{c}\text { E-plane/H-plane } \\
\left(d_{y}=0 \mathrm{~mm}\right)\end{array}$ & $\begin{array}{c}\text { E-plane/H-plane } \\
\left(d_{y}=3 \mathrm{~mm}\right)\end{array}$ & & $d_{y}=0 \mathrm{~mm}$ & $d_{y}=3 \mathrm{~mm}$ & \\
\hline 12 & $72 / 71.6$ & $72.6 / 70.8$ & $70.5 / 65.8$ & 8.16 & 8.36 & 7.47 \\
\hline 15 & $67 / 66.3$ & $60.9 / 61.7$ & $60.6 / 64.2$ & 8.95 & 9.76 & 8.7 \\
\hline 18 & $62.8 / 62.5$ & $60.9 / 57.7$ & $68.6 / 65.1$ & 9.73 & 9.95 & 9.36 \\
\hline
\end{tabular}

$d_{y}=0 \mathrm{~mm}$ represents the situation that the width of the brim is equal to the width of the waveguide.

radiation patterns versus different $d$. It can be observed that the metallic brims introduced here can suppress the back radiation of the waveguide antenna. According to Figure 6, the back radiation has been significantly suppressed when $d$ increased from 0 to $10 \mathrm{~mm}$. Moreover, the back radiation levels can be reduced to below $-20 \mathrm{~dB}$ across the whole $\mathrm{Ku}$ band when $d$ takes $10 \mathrm{~mm}$. This level of back radiation is very comparable to that reported in $[6,7,8,9]$ with a ground plane. However, in this case the beamwidth of the far-field pattern in E-plane is wider than that in H-plane. Since the equal Eand $\mathrm{H}$-plane radiation patterns are the main concentration in this paper, $d$ is finally chosen as $2.7 \mathrm{~mm}$. In this case, the back radiation level of the proposed antenna is less than $-11 \mathrm{~dB}$ within the whole Ku-band.

\section{Results and Discussions}

For numerical characterization, the proposed antenna is simulated using commercial software Ansoft HFSS. For verification, a prototype of the antenna is fabricated (see Figure
1) and measured using an Agilent N5230C network analyzer. The measured VSWR is also shown in Figure 3 and is in reasonable agreement with the simulated one. Measured result implies that the impedance bandwidth (VSWR $<1.4$ ) covers the whole Ku-band. Therefore, the matching performance of the waveguide antenna across the operating bandwidth has been improved dramatically by the introduction of the stepped ladders with appropriate dimensions.

The antenna radiation patterns are measured through the far-field measurement system in an anechoic chamber. Figure 7 illustrates the simulated and measured radiation patterns. As can be seen, the proposed antenna presents nearly identical beamwidths in E- and H-planes. Both simulated and measured results indicate that the cross-polarization (normalized to the copolarization) is considerably low in the entire frequency range. Furthermore, the backward radiation levels are less than $-11 \mathrm{~dB}$ across the entire operating band and are smaller in the upper frequency band than those in the lower frequency band. It is worth mentioning that the simulated cross-polarization levels are all less than $-35 \mathrm{~dB}$ 


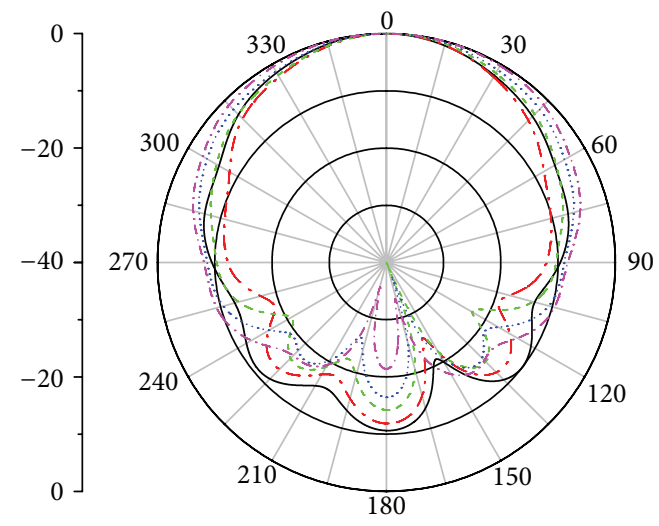

(a) E-plane

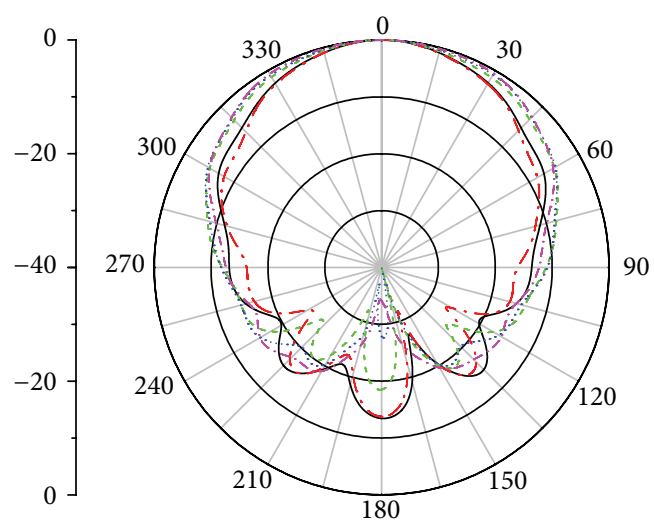

(c) E-plane

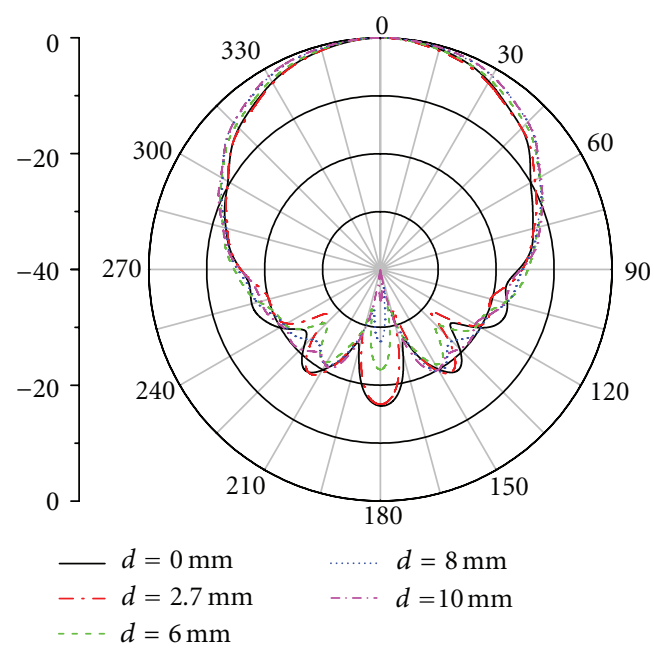

(e) E-plane

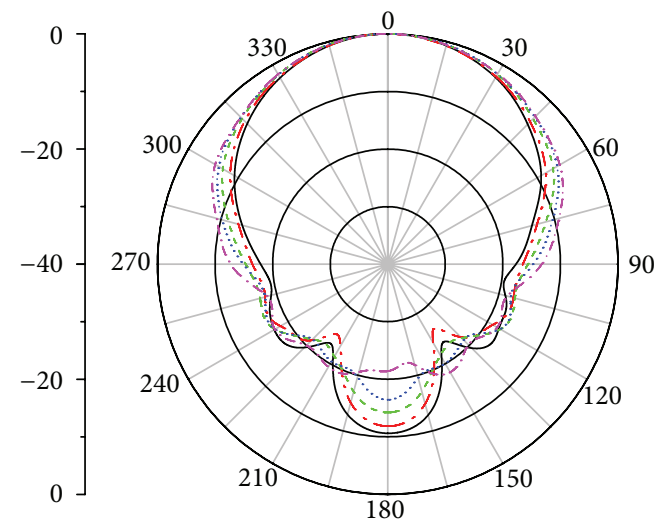

(b) H-plane

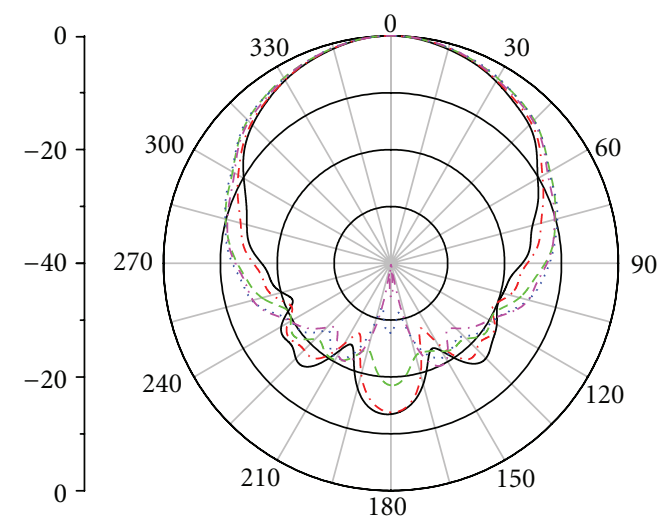

(d) H-plane

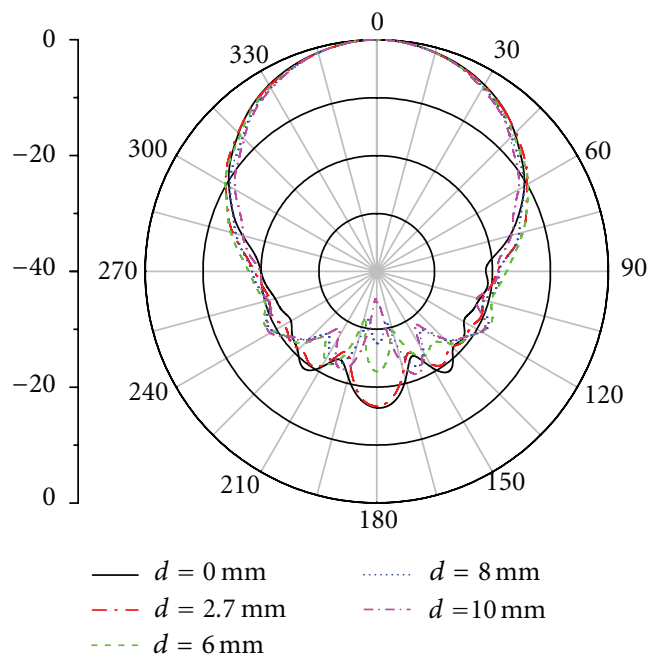

(f) H-plane

FIGURE 6: Study of variations in radiation patterns versus the changes of lengths of metallic brims at 12, 15, and $18 \mathrm{GHz}$, respectively. (a)-(b) E-plane and H-plane at $12 \mathrm{GHz}$, (c)-(d) E-plane and $\mathrm{H}$-plane at $15 \mathrm{GHz}$, and (e)-(f) E-plane and H-plane at $18 \mathrm{GHz}$.

while the measured ones are less than $-25 \mathrm{~dB}$ across the entire operating frequencies. The relatively large cross-polarization levels in measurements may be attributable to three factors: the first factor is the received weak signal which is on the order of the system noise and this is especially true for measuring the very low cross-polarization; the second factor is the large cross-polarization that is inherently induced by the transmitting horn, and this is especially true for the doubleridged horn which is used for measurement; the third factor is the tolerances of the pose for the transmitting and measured antennas which are impossible to be placed rigorously orthogonal. The tolerances can be reduced; however, they are 


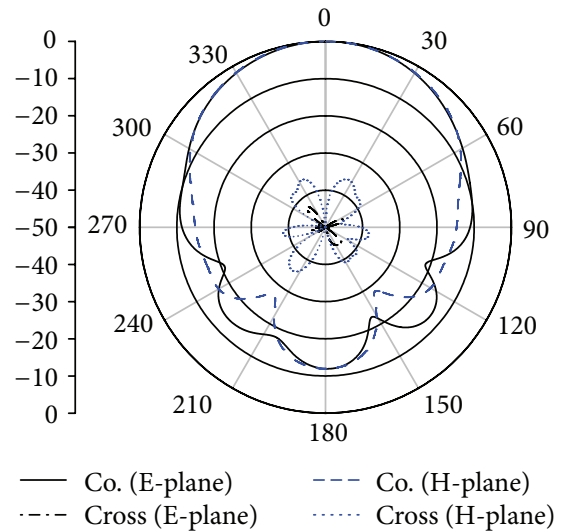

(a) $12 \mathrm{GHz}$

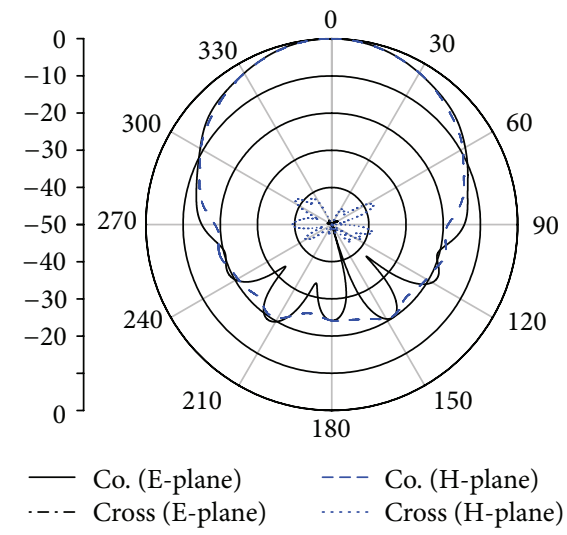

(c) $18 \mathrm{GHz}$

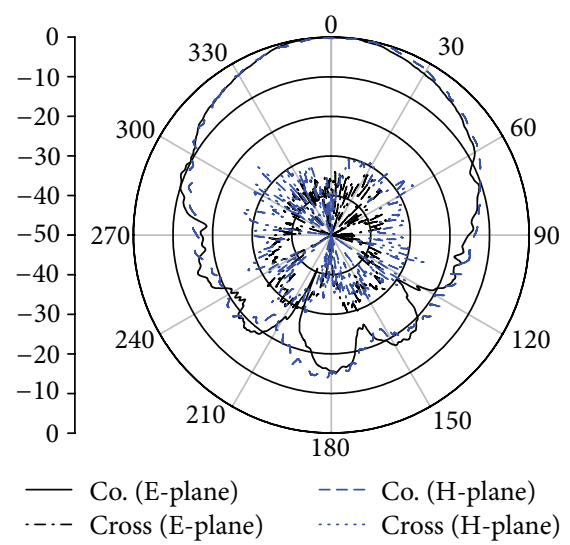

(e) $15 \mathrm{GHz}$

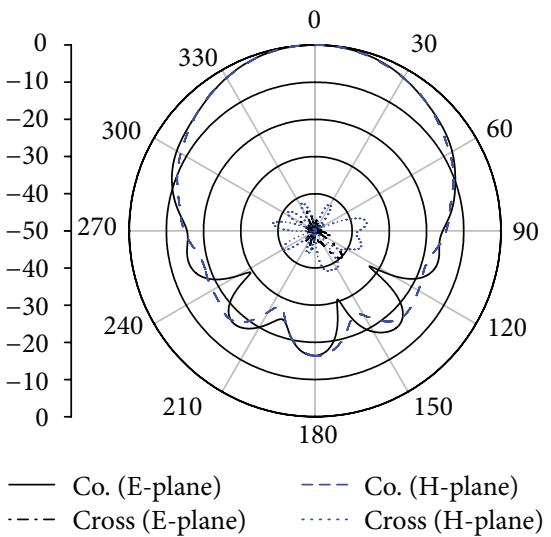

(b) $15 \mathrm{GHz}$

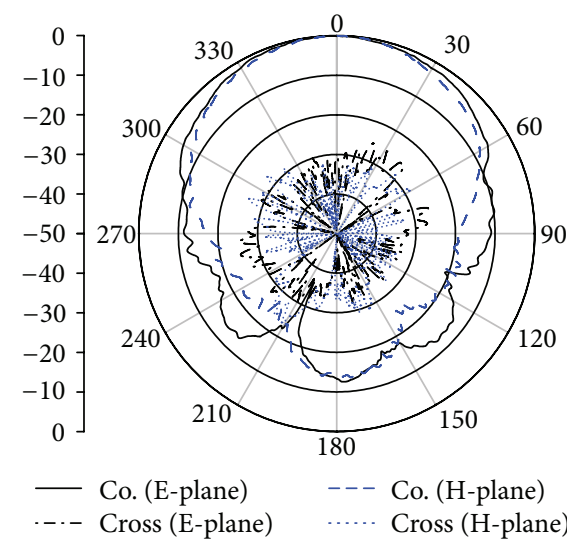

(d) $12 \mathrm{GHz}$

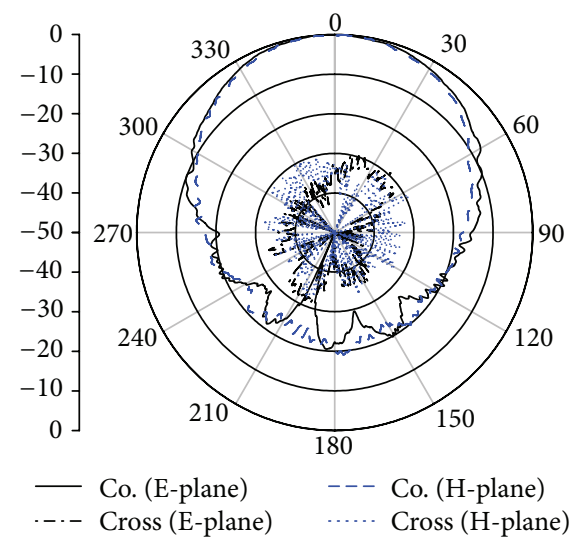

(f) $18 \mathrm{GHz}$

Figure 7: Simulated (a)-(c) and measured (d)-(f) radiation patterns of the proposed antenna at 12, 15, and $18 \mathrm{GHz}$, respectively.

hard to be eliminated. The detailed antenna performances including the gains and $3-\mathrm{dB}$ beamwidths for both $\mathrm{E}$ - and $\mathrm{H}$-planes at 12,15 , and $18 \mathrm{GHz}$ are summarized in Table 1. Clearly, the 3-dB beamwidth of the proposed antenna varies within 10 degrees across the observed frequency range, and the gain varies less than $2 \mathrm{~dB}$ when the frequency changes from 12 to $18 \mathrm{GHz}$. Therefore, the antenna exhibits a stable performance.

\section{Conclusion}

A novel open-ended rectangular waveguide antenna has been proposed, which features wide bandwidth, low crosspolarization, moderate backward radiation, and uniform pattern bandwidth (almost identical E- and H-plane patterns) across the whole Ku-band. Furthermore, the proposed antenna exhibits a simple structure and is conceptually easy 
to design. Hence, it can be widely employed as an efficient feed for circular-aperture antennas to obtain the desired pencil-beam radiation patterns.

\section{Acknowledgments}

This work is supported in part by the National Science Foundation of China under Grants nos. 60990320, 60990324, 61138001, and 60921063, the National High Tech (863) Projects under Grants nos. 2011AA010202 and 2012AA030702, and the 111 Project under Grant no. 111-2-05. M. Q. Qi would like to thank the anonymous reviewers for their constructive comments.

\section{References}

[1] Z. F. Liu, P. S. Kooi, L. W. Li, M. S. Leong, and T. S. Yeo, "A method for designing broad-band microstrip antennas in multilayered planar structures," IEEE Transactions on Antennas and Propagation, vol. 47, no. 9, pp. 1416-1420, 1999.

[2] K. F. Tong, K. M. Luk, K. F. Lee, and R. Q. Lee, "A broad-band Uslot rectangular patch antenna on a microwave substrate," IEEE Transactions on Antennas and Propagation, vol. 48, no. 6, pp. 954-960, 2000.

[3] K. M. Luk, C. L. Mak, Y. L. Chow, and K. F. Lee, "Broadband microstrip patch antenna," Electronics Letters, vol. 34, no. 15, pp. 1442-1443, 1998.

[4] Y. J. Cho, K. H. Kim, D. H. Choi, S. S. Lee, and S. O. Park, "A miniature UWB planar monopole antenna with $5-\mathrm{GHz}$ band-rejection filter and the time-domain characteristics," IEEE Transactions on Antennas and Propagation, vol. 54, no. 5, pp. 1453-1460, 2006.

[5] R. Zaker, C. Ghobadi, and J. Nourinia, "Novel modified UWB planar monopole antenna with variable frequency band-notch function," IEEE Antennas and Wireless Propagation Letters, vol. 7, pp. 112-114, 2008.

[6] A. Clavin, "A new antenna feed having equal E- and H-plane patterns," IRE Transactions on Antennas and Propagation, vol. 2, no. 3, pp. 113-119, 1954.

[7] A. Clavin, D. A. Huebner, and F. J. Kilburg, "An improved element for use in array antennas," IEEE Transactions on Antennas and Propagation, vol. 22, no. 4, pp. 521-526, 1974.

[8] K. M. Luk and H. Wong, "A new wideband unidirectional antenna element," International Journal of Microwave and Optical Technology, vol. 1, no. 1, pp. 35-44, 2006.

[9] L. Siu, H. Wong, and K. M. Luk, "A dual-polarized magnetoelectric dipole with dielectric loading," IEEE Transactions on Antennas and Propagation, vol. 57, no. 3, pp. 616-623, 2009.

[10] B. Q. Wu and K. M. Luk, "A broadband dual-polarized magnetoelectric dipole antenna with simple feeds," IEEE Antennas and Wireless Propagation Letters, vol. 8, pp. 60-63, 2009.

[11] B. Q. Wu and K. M. Luk, "A magneto-electric dipole with a modified ground plane," IEEE Antennas and Wireless Propagation Letters, vol. 8, pp. 627-629, 2009.

[12] L. Ge and K. M. Luk, "A low-profile magneto-electric dipole antenna," IEEE Transactions on Antennas and Propagation, vol. 60, no. 4, pp. 1684-1689, 2012.

[13] B. M. Thomas, "Design of corrugated conical horns," IEEE Transactions on Antennas and Propagation, vol. 26, no. 2, pp. 367-372, 1978.
[14] F. Hyjazie and R. Paknys, "On the radiation from a large, openended waveguide," IEEE Antennas and Propagation Magazine, vol. 44, no. 6, pp. 98-100, 2002.

[15] K. T. Selvan, "Studies on the classical gain approximations and the aperture-reflection coefficient of rectangular waveguide antennas," IEEE Transactions on Electromagnetic Compatibility, vol. 40, no. 4, pp. 343-347, 1998. 

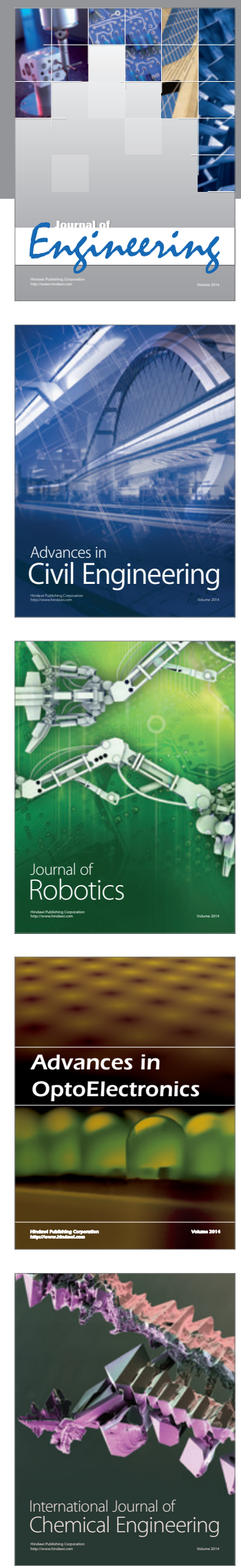

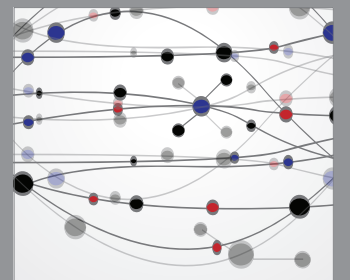

The Scientific World Journal
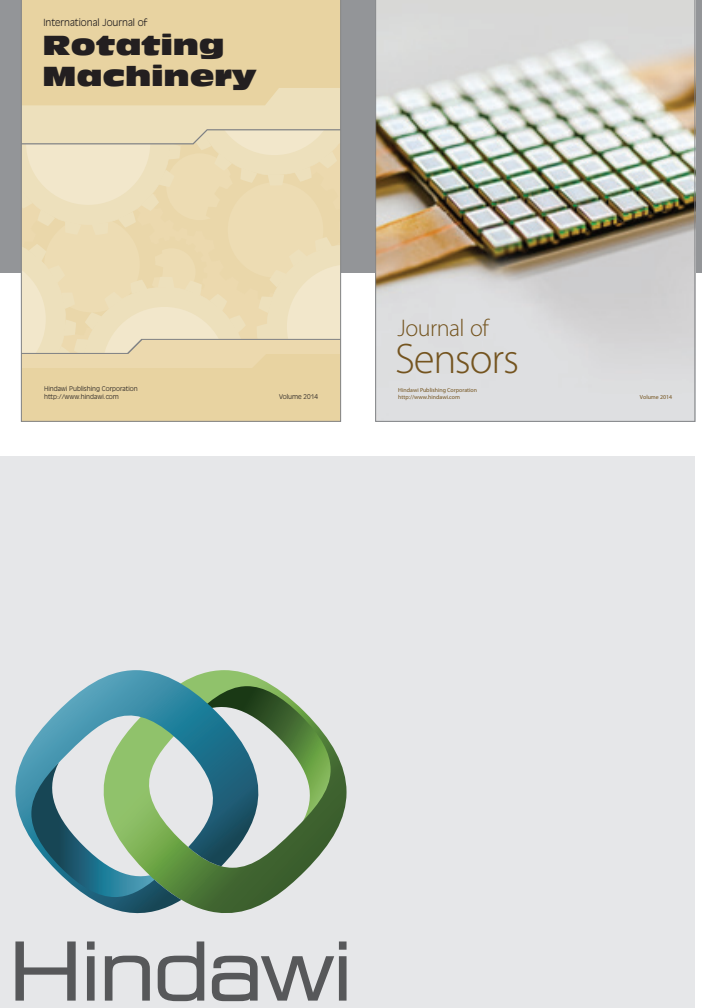

Submit your manuscripts at http://www.hindawi.com
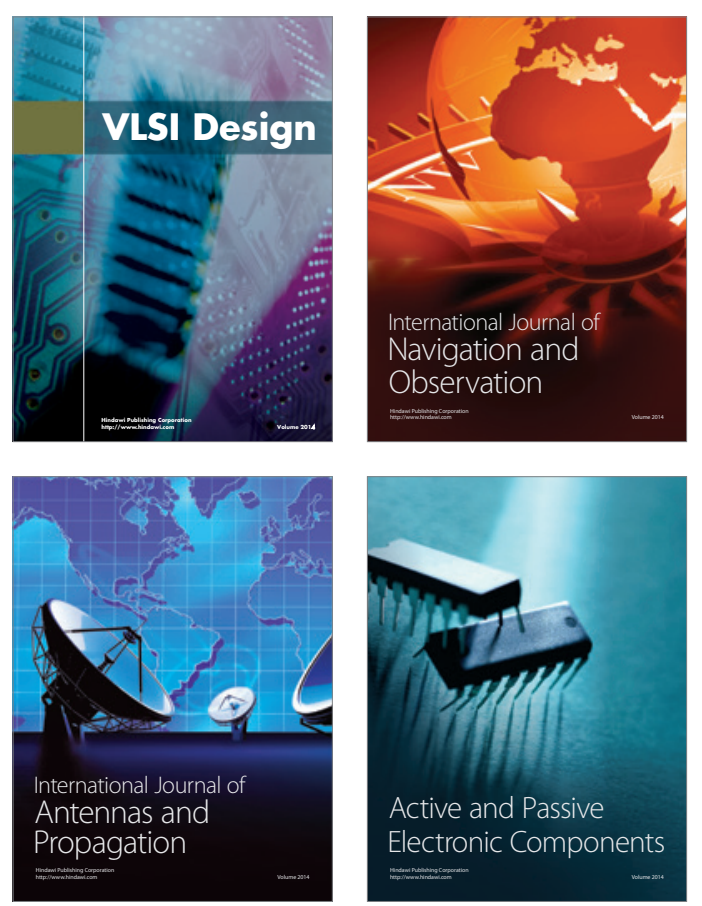
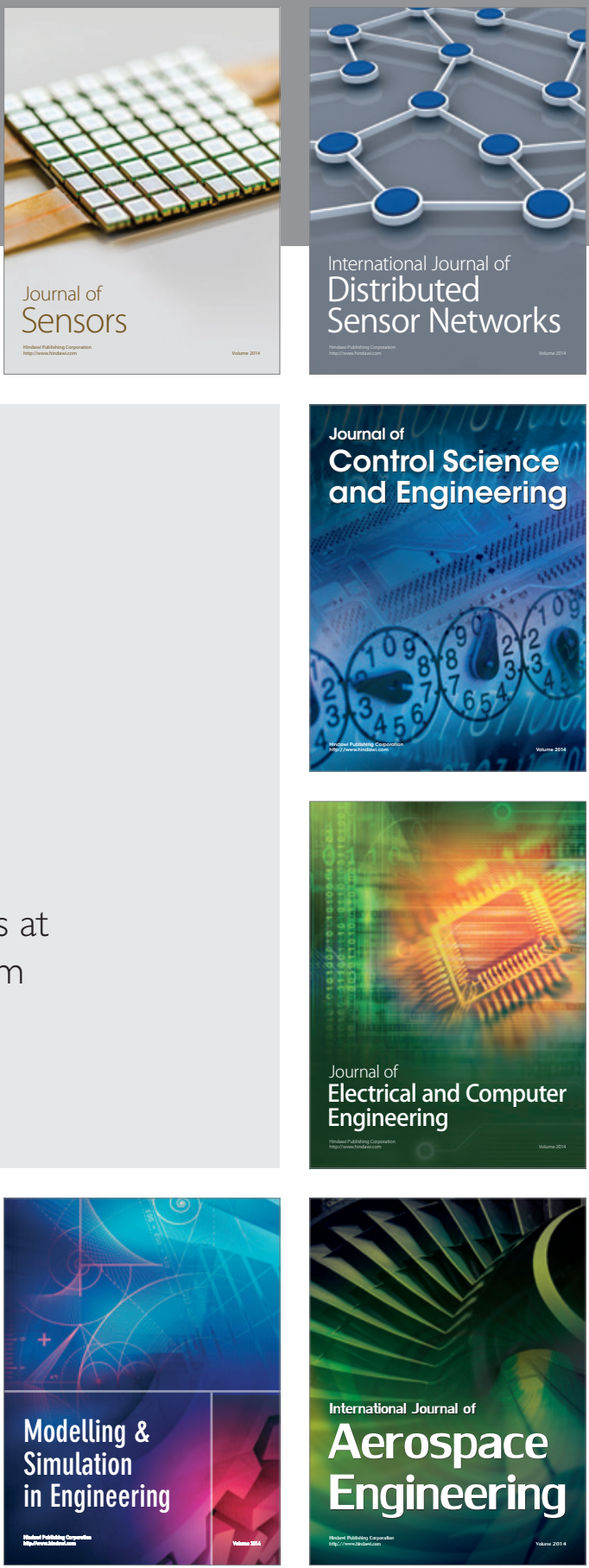

Journal of

Control Science

and Engineering
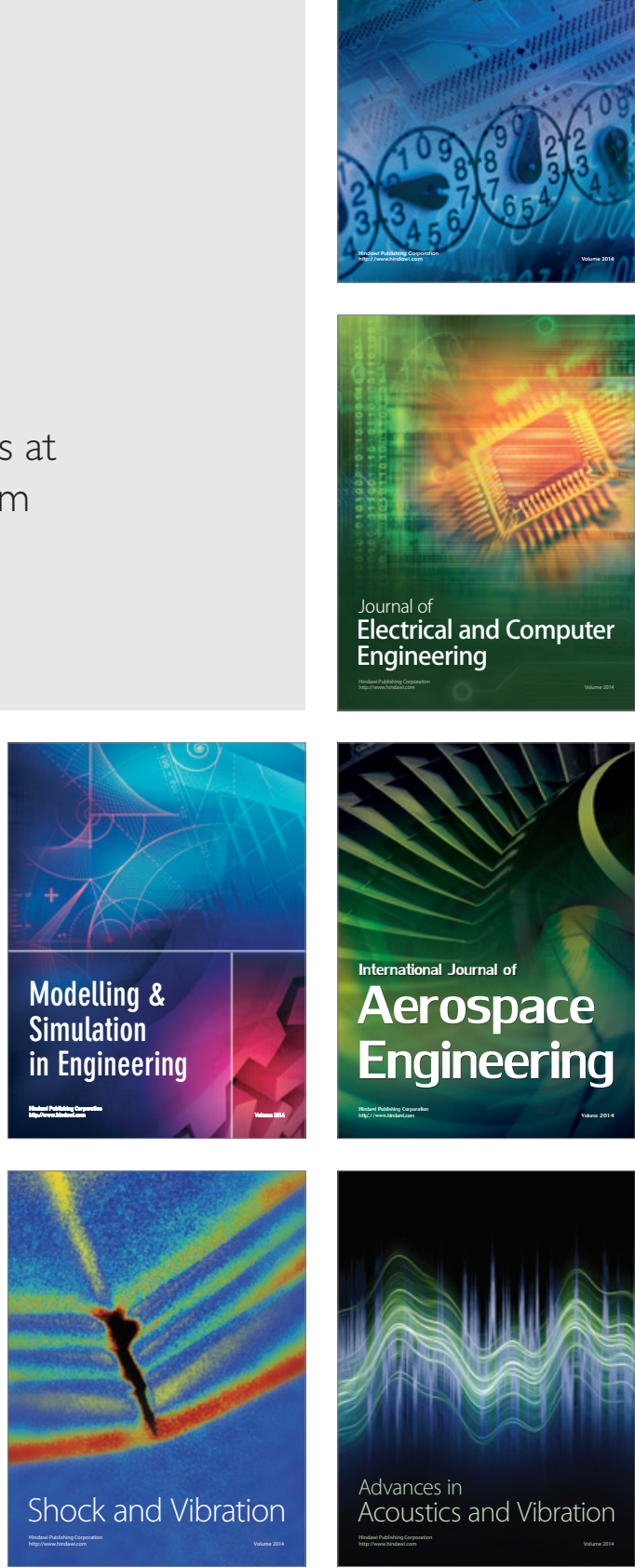